\title{
Pattern of Recurrence in Radically Treated Triple Negative Breast Cancer Patients
}

\author{
Aravindh Sivanandan Anand ${ }^{*}$, Annie Joseph ${ }^{1}$, Antony Stanley ${ }^{2}$ \\ ${ }^{1}$ Department of Radiation Oncology, Government Medical College, Trivandrum, India \\ ${ }^{2}$ Achutha Menon Centre, SCTIMST, Trivandrum, India \\ Email: *anandrt2006@yahoo.com
}

How to cite this paper: Anand, A.S., Joseph, A. and Stanley, A. (2021) Pattern of Recurrence in Radically Treated Triple Negative Breast Cancer Patients. Journal of Cancer Therapy, 12, 611-620.

https://doi.org/10.4236/jct.2021.1211053

Received: October 13, 2021

Accepted: November 16, 2021

Published: November 19, 2021

Copyright $\odot 2021$ by author(s) and Scientific Research Publishing Inc. This work is licensed under the Creative Commons Attribution International License (CC BY 4.0).

http://creativecommons.org/licenses/by/4.0/

\begin{abstract}
Background: In India approximately $20 \%$ of the patients with breast cancer are triple receptor negative. Owing to the aggressive nature and shorter disease-free survival judicious follow up and identification of failure pattern will benefit the patient. Similar studies have been conducted among non-Hispanic population and in China. This study aims to identify failure pattern in radically treated breast cancer patients who are triple receptor negative among Indian population. Methods: This prospective observational study was conducted in the Department of Radiation Oncology, a tertiary cancer centre in Kerala, India. The objective was to record the pattern of recurrence among triple negative breast cancer patients who completed their planned radical treatment. 171 patients with triple negative breast cancer were included in the study. Patients who completed the planned radical treatment were kept under regular follow up. Details of clinical examination and investigations during the follow up were recorded periodically. Results: Out of 171 patients 30 patients had a recurrence of disease. Median age of the population was 53 years. Among the 30 patients who had a recurrence, 16 patients (53\%) had systemic relapse and 14 patients (47\%) had locoregional relapse. Lung was found to be the most common site of distant metastasis (37\%). Ipsilateral chest wall was found to be the most common site of locoregional relapse (50\%). 6 months disease free survival was found to be $91.8 \%$ and 1-year disease free survival was found to be $70.2 \%$. Conclusion: Among radically treated triple negative breast cancers relapses, systemic recurrence was more than locoregional recurrences.
\end{abstract}

\section{Keywords}

Triple Negative Breast Cancer, Recurrence, Pattern of Failure, Locoregional, Distant Metastasis 


\section{Introduction}

Breast cancer is the most common type of cancer among women in the US with the incidence rate of $12.5 \%$. This disease is the primary cause of mortality among women aged 45 - 55 years and is the second leading cause of cancer-induced death. The incidence of breast cancer is almost 1 -in- 8 women, requiring complete tissue removal, chemotherapy, radiotherapy, and hormone therapy most of the time [1].

Basal-like tumours in general lack hormone receptors and HER2; thus, the majority of these tumors are also called triple-negative breast cancer (TNBC). Currently there is no molecular-based targeted therapy for TNBC, and unfortunately only approximately $20 \%$ of these tumors respond well to standard chemotherapy. Thus, developing improved treatments for TNBC is one of the highest priorities of current breast cancer research. By defining basal-like cancers either in terms of expression of basal immunohistochemical markers or a basal-like RNA expression profile, studies are consistent in their morphological description of this subtype as high grade, largely ductal, but occasionally either metaplastic, atypical or typical medullary, or adenoid cystic cancers [1].

Hormone receptor-negative breast cancers are a heterogeneous group of breast cancers that are generally thought to be aggressive with poor prognosis and with fewer cancer prevention and treatment strategies compared with tumors expressing hormone receptor. In addition, HER2-negative tumors lack the benefit of specific therapy that targets this protein (e.g., trastuzumab-based therapy). Therefore, treatment options for these triple-negative tumors are more limited [2]. The prognostic significance of determining oestrogen receptor (ER), progesterone receptor (PR), and human epidermal growth factor 2 expression (HER2) has been studied by various groups [3].

Triple-negative breast cancers (TNBCs) are characterized by a high proliferation rate and increased aggressiveness compared with other subtypes. Because endocrine and HER2-targeted therapies cannot be offered, conventional cytotoxic chemotherapy followed by adjuvant RT is the standard of care for patients with TNBC [4]. Studies evaluating pathological response of triple negative breast cancer with neoadjuvant chemotherapy are being conducted in several parts of the world [5] [6].

The pattern of spread is distinct from that for hormone receptor-positive tumours: TNBC has a greater propensity for brain and lung metastases, and a lower prevalence of bone metastases. Compared with those having hormone receptor-positive, her2-negative tumours had a worser OS, the difference being most pronounced in the first 2 years [7].

Although triple negative tumors have been reported to be more aggressive, there are limited long-term clinical data evaluating outcome as a function of this classification. Even though clinicians routinely use conventional ER, PR, and HER2/neu status to classify patients as triple negative for clinical decision making, there are no large databases that have evaluated locoregional control in 
conservatively managed patients. Studies are being conducted to evaluate disease failure patterns and overall survival (OS) of women with TNBC, who undergo breast conservation treatment [8]. The baseline prognosis for patients with basal-like breast cancer is poor in the absence of therapy, so it is critical to identify the best therapeutic targets for these patients. The chemotherapy benefit is high, but there are many promising new targets and approaches for this group, including PARP inhibitors, a potential carboplatin benefit, angiogenesis inhibitors, and HER-1-RAS-mitogen-activated protein kinase/extracellular signal-related kinase pathway inhibitors [9].

The rationale behind conducting this study is to address the pattern of recurrence, i.e., any organ predilection and survival outcomes which will benefit our patients for planning follow up and early detection of metastasis.

\section{Methods}

This study was conducted in the Department of Radiotherapy and Oncology of Government Medical College, Trivandrum in State of Kerala, India from December 2017 to December 2019. This institution is a tertiary cancer centre of the State. This study was approved by the scientific Institutional Review Board and by the Human ethical committee.

The primary objective is to study the pattern of recurrence in radically treated TNBC patients whether locoregional (breast or nodal pattern) or systemic (organ predilection). The secondary objective is to estimate disease free survival in radically treated triple negative breast cancer patients.

All consecutive patients with biopsy and IHC proven diagnosis of triple negative breast receptor status, who completed the planned radical treatment (chemotherapy, surgery and radiotherapy if indicated) and willing to participate in the study were included.

Female patients in the age group 18 to 75 years, with ECOG performance status of 0 to 2 and stage I - III were included in the study. Metastatic disease at presentation, patients with any other cancers, patients with medical contraindications for chemotherapy and radiotherapy, and poor performance status patients (ECOG 3, 4) were excluded.

After obtaining an informed consent, data was collected using a structured proforma, which detailed patient characteristics, clinical examination findings, histopathology report, and treatment done. Nature of the study was explained to each patient in their respective local language, Malayalam.

Patients were observed prospectively, and no alteration is done in the standard ongoing treatment for the purpose of study. Surgery followed by adjuvant chemotherapy and radiation as per standard current guidelines were administered. Patients received four 3-week cycles of epirubicin $90 \mathrm{mg} / \mathrm{m}^{2}$ or doxorubicin $60 \mathrm{mg} / \mathrm{m}^{2}$ and cyclophosphamide $600 \mathrm{mg} / \mathrm{m}^{2}$ followed by the combination of four 3-week cycles of docetaxel $75 \mathrm{mg} / \mathrm{m}^{2}$ or other standard chemotherapy schedules according to performance status and body mass index. Patients received 
local radiation treatment either as $50 \mathrm{~Gy} / 25 \mathrm{fr}$ or $40 \mathrm{~Gy} / 15 \mathrm{fr}$. Clinical examination after each cycle and after completion of treatment was done for clinical response and toxicity manifestations.

Suspected cases of Loco regional relapses were confirmed with FNAC/biopsy. Investigations were done according to patient symptoms and systemic recurrences confirmed by radiological investigations as per standard guidelines.

Data analysis was done with the help of Excel 2010 and SSPS 18 statistical software. Site of recurrence, organ involved, date of treatment completion and disease-free survival rates has been entered in Excel 2010 work sheet for each variable. Pattern of failure was noted and organ with higher predilection for systemic metastasis was identified. 1 year disease free survival rates and 6-month disease free survival rates were estimated.

\section{Results}

A total of 171 patients satisfying the selection criteria were included in this study (Tables 1-5, Figure 1).

Median age of the population under study was found to be 53 years. Majority of patients $(>90 \%)$ presented with a lump in the breast. Only less than $10 \%$ patients presented with symptoms such as nipple retraction, nipple discharge and pain in breast. More than $60 \%$ patients included in the study had a performance status of 1 and hence were able to complete the planned radical treatment. $\mathrm{Pa}$ tients with poor performance status were excluded from the study. Most of the patients included in the study were married and had children. Only 9 out 141 patients $(6 \%)$ who did not have a recurrence were nulliparous. In this study majority of the women were of the post-menopausal group (>70\%).

In this study 107 patients (61\%) belonged to the early T stage (T1 and T2) and 64 patients (38\%) belonged to the advanced T stage (T3 and T4).

Out of 171 patients included in the study 75 patients (44\%) were node negative. 52 patients (30\%) had N1 disease, 28 patients (16\%) had N2 disease, and 16 patients (9\%) had N3 disease.

In this study 11 patients (6\%) presented in Stage I, 88 patients (51\%) presented in Stage II, and 72 patients (42\%) presented in Stage III. More than 95\% of patients had the histological subtype infiltrating ductal carcinoma and the rest

Pattern of Recurrence
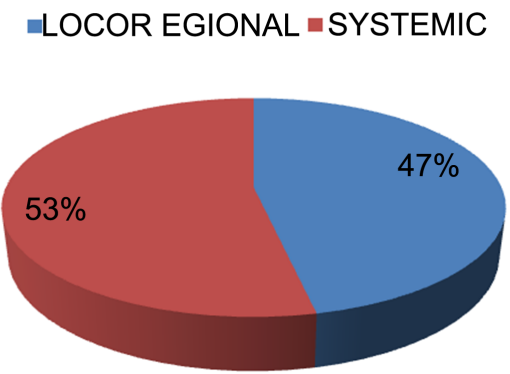

Figure 1. Pattern of recurrence. 
Table 1. Base line characteristics of the patients.

\begin{tabular}{|c|c|c|}
\hline Variable & Frequency & Percentage \\
\hline \multicolumn{3}{|l|}{ Age of patient } \\
\hline$<41$ & 21 & 12.3 \\
\hline $41-50$ & 61 & 35.6 \\
\hline $51-60$ & 34 & 19.8 \\
\hline $61-70$ & 25 & 14.6 \\
\hline$>70$ & 10 & 5.8 \\
\hline \multicolumn{3}{|l|}{ Symptoms } \\
\hline Lump & 162 & 94.7 \\
\hline Nipple retraction & 5 & 2.9 \\
\hline Others & 4 & 2.3 \\
\hline \multicolumn{3}{|l|}{ ECOG performance status } \\
\hline 0 & 51 & 29.8 \\
\hline 1 & 102 & 59.6 \\
\hline 2 & 18 & 10.5 \\
\hline \multicolumn{3}{|l|}{ Parity } \\
\hline Nulliparous & 9 & 5.3 \\
\hline One child & 18 & 10.5 \\
\hline Two children & 101 & 59.1 \\
\hline More than 2 & 43 & 25.1 \\
\hline \multicolumn{3}{|l|}{ Menopausal status } \\
\hline Pre-menopausal & 46 & 26.9 \\
\hline Post-menopausal & 114 & 66.6 \\
\hline Post Hysterectomy & 11 & 6.4 \\
\hline \multicolumn{3}{|l|}{ Co morbidities } \\
\hline Hypertension & 22 & 12.8 \\
\hline Diabetes mellitus & 19 & 11.1 \\
\hline Dyslipidaemia & 2 & 1.1 \\
\hline Hypothyroidism & 11 & 6.4 \\
\hline Multiple co morbidities & 27 & 15.7 \\
\hline No comorbidities & 90 & 52.6 \\
\hline
\end{tabular}

Table 2. Tumour characteristics.

\begin{tabular}{|c|c|c|c|c|}
\hline Variable & Frequency & $\%$ & Frequency & $\%$ \\
\hline 1) Location of tumour & $\begin{array}{l}\text { Recurrence } \\
\text { group }\end{array}$ & & $\begin{array}{c}\text { No recurrence } \\
\text { group }\end{array}$ & \\
\hline
\end{tabular}

a) Side of tumour

Left

19

63.3

74 


\section{Continued}

$\begin{array}{lllll}\text { Right } & 11 & 36.7 & 67 & 47.6\end{array}$

b) Quadrant of tumour

Upper outer quadrant

83.3

113

80.1

Upper inner quadrant

6.7

6

Lower outer quadrant

6.7

6

Lower inner quadrant

$$
3.3
$$

3

Paracentral

0

13

9.2

2) Staging of tumour

a) $\mathrm{T}$ stage

$\begin{array}{ccccc}\text { T1 } & 2 & 6.6 & 13 & 9.2 \\ \text { T2 } & 15 & 50 & 77 & 54.6 \\ \text { T3 } & 10 & 33.3 & 36 & 25.5 \\ \text { T4 } & 3 & 10 & 15 & 10.6\end{array}$

b) N stage

N0
N1
N2
N3

c) Composite stage

Stage I
Stage II
Stage III

0

0

11

7.8

14

46.6

74

52.4

16

53.3

56

39.7

\section{3) Pathological features}

a) Histological subtypes

Infiltrating ductal carcinoma

Metaplastic carcinoma

Others

b) Grade of tumour

1
2
3

c) Lymphovascular invasion

\begin{tabular}{ccccc}
1 & 1 & 3.3 & 7 & 4.9 \\
2 & 14 & 46.6 & 79 & 56 \\
3 & 15 & 50 & 55 & 39 \\
ascular invasion & & & & \\
Yes & 8 & 26.7 & 16 & 11.3 \\
No & 22 & 73.3 & 125 & 88.6 \\
\hline
\end{tabular}

$\begin{array}{cccc}29 & 96.6 & 128 & 90.7 \\ 1 & 3.3 & 8 & 5.6 \\ 0 & 0 & 5 & 3.5\end{array}$

3.5

46.1

32.6

14.2

7.1 


\section{Continued}

d) Multifocality

\begin{tabular}{ccccc} 
Yes & 1 & 3.3 & 4 & 2.8 \\
No & 29 & 96.7 & 137 & 97.2 \\
\hline
\end{tabular}

Table 3. Treatment characteristics.

\begin{tabular}{ccccc}
\hline Variable & Frequency & $\%$ & Frequency & $\%$ \\
\hline 1) Type of surgery & & & & \\
BCS + AC & 6 & 20 & 1 & 0.7 \\
MRM & 24 & 80 & 139 & 98.5 \\
Toilet mastectomy & 0 & 0 & 1 & 0.7
\end{tabular}

\section{2) Chemotherapy}

a) Schedule

$\begin{array}{ccccc}\text { Neo-adjuvant } & 10 & 33.3 & 22 & 15.6 \\ \text { Adjuvant } & 20 & 66.6 & 119 & 84.3\end{array}$

b) Type of chemotherapy

$\begin{array}{ccccc}\mathrm{AC}^{*} 4>\mathrm{T} * 4 & 22 & 73.3 & 92 & 65.2 \\ \mathrm{FAC}{ }^{*} 6 & 3 & 10 & 19 & 13.4 \\ \mathrm{FEC}{ }^{*} 6 & 3 & 10 & 21 & 14.9 \\ \mathrm{TAC} * 6 & 2 & 6.7 & 9 & 6.3\end{array}$

3) Radiation

$\begin{array}{ccccc}\text { Yes } & 28 & 93.3 & 105 & 74.5 \\ \text { No } & 2 & 6.7 & 36 & 25.5\end{array}$

\section{4) Radiation schedule}

Conventional fractions

Hypo fractionated

Table 4. Site of relapse.

\begin{tabular}{ccc}
\hline Variable & Frequency & $\%$ \\
\hline 1) Locoregional relapse & & \\
Ipsilateral breast & 4 & 28.6 \\
Ipsilateral chest wall & 7 & 50 \\
Ipsilateral axilla & 2 & 14.2 \\
Contra lateral breast & 1 & 7.1
\end{tabular}

2) Distant metastasis

Lung

6

37.5 
Continued

\begin{tabular}{ccc}
\hline Liver & 2 & 12.5 \\
Brain & 4 & 25 \\
Bone & 2 & 12.5 \\
More than one organ & 2 & 12.5 \\
\hline
\end{tabular}

Table 5. Disease free survival.

\begin{tabular}{ccc}
\hline Time & Disease free number & DFS \% \\
\hline 6 months DFS & 157 & 91.8 \\
12 months DFS & 120 & 70.2 \\
\hline
\end{tabular}

of the patients had metaplastic carcinoma and other histological variants. Majority of the tumour were of grade 3 (50\%) among those 30 patients who had recurrence. 24 patients (14\%) showed lympho-vascular space invasion in the histology.

163 patients underwent modified radical mastectomy, and 7 patients underwent breast conservation surgery, and 1 patient underwent toilet mastectomy. 32 patients (19\%) took neoadjuvant chemotherapy, and 139 patients (81\%) took adjuvant chemotherapy.

The most used chemotherapy regimen among our study population was 4 cycles of Adriamycin and cyclophosphamide followed by 4 cycles of taxane which is the standard treatment regimen for triple negative breast cancer according to literature. 133 patients (77\%) received adjuvant radiation treatment. 30 patients $(17 \%)$ out of 171 patients with triple negative breast cancer had recurrence of disease.

Among 30 patients who recurred 14 patients (47\%) had locoregional recurrence and 16 patients had systemic recurrence with the most common organ with metastatic deposits being lung ( 6 patients) followed by brain ( 4 patients), liver and bone ( 2 patients each)/of the locoregional relapses, it was found that ipsilateral chest wall had a higher propensity to failure following MRM and that in those patients who underwent BCS the failure was in ipsilateral breast itself. 6 months disease free survival of the study population was found to be $91.8 \%$ and 1 year DFS was found to be $70.2 \%$.

\section{Discussion}

Triple negative subtype lacks any specific target and currently is subject to vigorous investigations to improve its outcome. TNBC also is a biologically extremely heterogeneous group of breast cancer, with a high likelihood of recurrence during the first 2 - 3 years after diagnosis.

The incidence of recurrence in this study which is $17.5 \%$ is more than that seen in the study conducted by Abdulkarim et al. [4] which is $10 \%$. The incidence of systemic metastasis (53\%) is more than the incidence of locoregional 
recurrence (47\%). This observation is similar to the study conducted by Prasad et al. [10]. Like other studies, lung metastasis is the most common site for systemic metastases and ipsilateral chest wall the site for loco-regional recurrence. The 6 months disease free survival of $91.8 \%$ and 1 year disease free survival of $70.2 \%$ is almost like the results obtained in various other studies conducted among Western and Asian population [5] [10]. Our study has its own limitations. There is lack of long term follow up and the sample size is small in our study.

\section{Conclusion}

Triple negative breast cancer constitutes a heterogeneous group of breast cancer patients who have an aggressive behaviour, the incidence of recurrence in radically treated triple negative breast cancer patients included in this study was found to be $17.5 \%$ of which local recurrence is the major share.

\section{Conflicts of Interest}

The authors declare no conflicts of interest regarding the publication of this paper.

\section{Ethical Approval}

The study was approved by the Institutional Human Ethics Committee of Government Medical College, Thiruvananthapuram, Kerala, India.

\section{References}

[1] Cleator, S., Heller, W. and Coombes, R.C. (2007) Triple-Negative Breast Cancer: Therapeutic Options. The Lancet Oncology, 8, 235-244. https://doi.org/10.1016/S1470-2045(07)70074-8

[2] Rakha, E.A., El-Sayed, M.E., Green, A.R., Lee, A.H.S., Robertson, J.F. and Ellis, I.O. (2007) Prognostic Markers in Triple-Negative Breast Cancer. Cancer, 109, 25-32. https://doi.org/10.1002/cncr.22381

[3] Wang, S.-L., Li, Y.-X., Song, Y.-W., Wang, W.-H., Jin, J., Liu, Y.-P., et al. (2011) Triple-Negative or HER2-Positive Status Predicts Higher Rates of Locoregional Recurrence in Node-Positive Breast Cancer Patients after Mastectomy. International Journal of Radiation Oncology, 80, 1095-1101. https://doi.org/10.1016/j.ijrobp.2010.03.038

[4] Abdulkarim, B.S., Cuartero, J., Hanson, J., Deschênes, J., Lesniak, D. and Sabri, S. (2011) Increased Risk of Locoregional Recurrence for Women with T1-2N0 Triple-Negative Breast Cancer Treated with Modified Radical Mastectomy without Adjuvant Radiation Therapy Compared with Breast-Conserving Therapy. Journal of Clinical Oncology, 29, 2852-2858. https://doi.org/10.1200/JCO.2010.33.4714

[5] Chen, V.E., Gillespie, E.F., Zakeri, K., Murphy, J.D., Yashar, C.M., Lu, S., et al. (2017) Pathologic Response after Neoadjuvant Chemotherapy Predicts Locoregional Control in Patients with Triple Negative Breast Cancer. Advances in Radiation Oncolo$g y, 2,105-109$. https://doi.org/10.1016/j.adro.2017.01.012

[6] Liedtke, C., Mazouni, C., Hess, K.R., André, F., Tordai, A., Mejia, J.A., et al. (2008) Response to Neoadjuvant Therapy and Long-Term Survival in Patients with Triple- 
Negative Breast Cancer. Journal of Clinical Oncology, 26, 1275-1281.

https://doi.org/10.1200/JCO.2007.14.4147

[7] Lebert, J.M., Lester, R., Powell, E., Seal, M. and McCarthy, J. (2018) Advances in the Systemic Treatment of Triple-Negative Breast Cancer. Current Oncology, 25, 142-150. https://doi.org/10.3747/co.25.3954

[8] Zaky, S.S., Lund, M., May, K.A., Godette, K.D., Beitler, J.J., Holmes, L.R., et al. (2011) The Negative Effect of Triple-Negative Breast Cancer on Outcome after BreastConserving Therapy. Annals of Surgical Oncology, 18, 2858-2865. https://doi.org/10.1245/s10434-011-1669-4

[9] Perou, C.M. (2011) Molecular Stratification of Triple-Negative Breast Cancers. The Oncologist, 16, 61-70. https://doi.org/10.1634/theoncologist.2011-S1-61

[10] Prasad, S., Efird, J.T., James, S.E., Walker, P.R., Zagar, T.M. and Biswas, T. (2016) Failure Patterns and Survival Outcomes in Triple Negative Breast Cancer (TNBC): A 15 Year Comparison of 448 Non-Hispanic Black and White Women. SpringerPlus, 5, Article No. 756. https://doi.org/10.1186/s40064-016-2444-6 